\title{
Alteration of $\beta$-D-Glucan from Edible Mushroom after Injection into Mouse Peritoneal Cavity
}

\author{
Yoshiaki Sone,* Satoaki ShiBATA, Etsu KishidA,** \\ and Akira MisAKI \\ Department of Food and Nutrition, Faculty of Human Life Science, \\ Osaka City University, Sumiyoshi-ku, Osaka 558, Japan
}

(Received March 4, 1994)

\begin{abstract}
Summary Tritium-labeled antitumor $\beta$-D-glucan derivative (T-labeled glucan) was prepared from the branched $\beta-1,3$-D-glucan of an edible mushroom, Volvariella volvacea, by its periodate oxidation followed by reduction with $\mathrm{NaBT}_{4}$. Twenty-three hours after T-labeled glucan had been injected into the mouse peritoneal cavity, about 3\% of the total radioactivity injected was found in the mouse serum. In spite of the fact that T-labeled glucan had no affinity to the anion-exchange column before injection, about $50 \%$ of the labeled $\beta$-D-glucan in the serum thus obtained was adsorbed onto the column. The labeled $\beta$-D-glucan fraction eluted from the column by salt gradient showed antitumor activity in vivo.

Key Words edible mushroom $\beta$-D-glucan, labeled $\beta$-D-glucan, antitumor activity, intraperitoneal injection
\end{abstract}

Recently, certain food constituents were found to have some physiological activities, and attracted attention as food additives for the development of human nutritional conditions or clinical applications. Among them, $\beta$-D-glucans, which are usually extracted from fruiting bodies of edible mushrooms, were shown to have immunomodulating activities (1). Since we found host-mediated tumor-growth inhibition activities in hot-alkaline extraction of an edible mushroom of Kikurage, Auricularia auricula-judae (2), we have studied the relationship between antitumor activities and the structural features of $\beta$-D-glucans extracted from edible mushrooms, such as Ganoderma lucidum (3) and Volvariella volvacea (4), from the viewpoints of some physiological effects of food constituents on human health.

In the course of our investigation on the mechanism of the antitumor action of the $\beta$-D-glucan isolated from an edible mushroom Volvariella volvacea $(5,6)$, we found antitumor activity in the mouse serum obtained 10 to $25 \mathrm{~h}$ after the intraperitoneal injection of the glucan. In this connection, Sasaki et al. (7) reported

* To whom reprint requests should be addressed.

** Present address: Department of Life and Health Sciences, Hyogo University of Teacher Education, Katoh-gun, Hyogo 673-14, Japan. 
that the sera from the mice which had been treated with the antitumor $\beta-(1 \rightarrow 3)$ D-glucan enhanced the macrophage-mediated cytotoxicity in vitro. These facts prompted us to trace the antitumor $\beta$-D-glucan and detect its alteration after its intraperitoneal injection using tritium-labeled antitumor $\beta$-D-glucan derivative.

\section{MATERIALS AND METHODS}

Antitumor $\beta$-D-glucan was isolated from the fruiting body of $V$. volvacea by the alkaline extraction described previously (5). Tritium (T)-labeled glucan was prepared as follows. The $\beta$-D-glucan $(30 \mathrm{mg})$ was oxidized with $0.5 \mathrm{mM} \mathrm{NaIO}$ at $5^{\circ} \mathrm{C}$ for $120 \mathrm{~h}$ in the dark. After oxidation, a small amount of ethylene glycol was added to decompose residual $\mathrm{IO}_{4}{ }^{-}$, and the solution was dialyzed against distilled water. To the $\mathrm{IO}_{4}{ }^{-}$oxidized glucan solution, $\mathrm{NaBT}_{4}(0.1 \mu \mathrm{Ci}$, Amersham International plc, England) was added for partial reduction with tritium and the solution was left overnight. The resulting partially reduced glucan was then treated with 10 $\mathrm{mg}$ of $\mathrm{NaBH}_{4}$ to complete the reduction. The T-labeled glucan was purified by chromatographies using ion-exchange and gel-permeation columns of an FPLC system (Pharmacia Biotech., Uppsala, Sweden). Glucan content was determined by the phenol-sulfuric method (8) using the native $\beta$-D-glucan as a standard.

Gel-permeation FPLC was carried out using a Superose $12 \mathrm{HR}$ column $(1 \times 30$ $\mathrm{cm})$ and $0.02 \mathrm{M}$ Tris-acetate buffer at $\mathrm{pH} 7.0$ as an elution buffer. Ion-exchange FPLC was done with Mono Q HR $5 / 5$ column $(0.5 \times 5 \mathrm{~cm})$ and elution buffer system of salt gradient from $0.02 \mathrm{M}$ Tris-acetate buffer, $\mathrm{pH} 7.2$ to the same buffer containing $1.0 \mathrm{M} \mathrm{NaCl}$.

Radioactivity was measured by an LKB liquid scintillation counter using a xylene-based counting cocktail (Scintisol, Wako Pure Chemical, Osaka, Japan). Colored solutions such as mouse serum were de-colored by the addition of one drop of $\mathrm{H}_{2} \mathrm{O}_{2}$, then mixed into the scintillation cocktail.

Antitumor activities of the fractions obtained by FPLC using Mono Q column were assayed in vivo by the method described previously (5). Each fraction was sterilized by passage through a membrane filter (Acrodisc, Gelman Sciences Inc., MI, U.S.A., $0.2 \mu \mathrm{m})$ and the aliquots $(0.1 \mathrm{ml})$ were injected intravenously (i.v.) into the tumor-bearing mice on 3 successive days from the 7 th day after Sarcoma 180 tumor-cells were implanted (average tumor volume was $0.8 \pm 0.2 \mathrm{ml}$ ) in ICRJcl mice (Japan Clea, Tokyo, Japan). The volume of Sarcoma 180 solid tumor was estimated by the following equation: tumor volume $(\mathrm{ml})=3.14 \times d_{1} d_{2}{ }^{2} / 6$, where $d_{1}$ is the length of major axis and $d_{2}$ is that of minor axis of the solid tumor. Tumor-growth inhibition ratio (\%) was calculated by the following equation: Inhibition ratio $(\%)=(A-B) \mid A \times 100$, where $A$ is the average tumor volume of the control group and $B$ is that of the treated group. 


\section{RESULTS AND DISCUSSION}

As reported previously (9), conversion of the glucosyl groups attached at O-6 of the 1,3-linked D-glucosyl residues of the antitumor $\beta$-D-glucan into the corresponding polyhydroxyl groups significantly enhanced the original antitumor activity. This fact makes it possible to prepare the tritium-labeled antitumor $\beta$-D-glucan derivative by the reduction of $\mathrm{IO}_{4}{ }^{-}$-oxidized glucan with $\mathrm{NaBT}_{4}$. After labeling procedures, the labeled glucan derivative was applied on a Mono-Q anion-exchange column for its purification. As shown in Fig. 1, the radioactive material was separated into two fractions. Because absorption in the ultraviolet region (UV) was detected in the fractions eluted by a salt gradient, the fractions not retained on the column were pooled, concentrated and subjected to gel-permeation chromatography for further purification. The fractions eluted at the void volume (molecular mass over $200 \mathrm{kDa}$ ) were pooled and concentrated. Specific activity of the purified labeled glucan was about $140 \times 10^{3} \mathrm{cpm} / \mathrm{mg}$ glucan. It should be noted that molecular weight and "neutrality" of the labeled glucan to Mono-Q anion exchange column did not change after standing at room temperature for more than $24 \mathrm{~h}$.

Twenty-three hours after intraperitoneal (i.p.) injection of the labeled glucan at a dose of $90 \mathrm{mg} / \mathrm{kg}$ mouse into normal mice, distribution of the labeled-glucan in the serum, in some organs (liver, kidney, spleen) and in the peritoneal cavity was determined. In this experiment, the sum total of the radioactivities found in mouse serum and in the organs reached to about $10 \%$ of the total radioactivity injected. Of the radioactivity found, about $30 \%$ was detected in the serum. The change of radioactivity level of the labeled $\beta$-glucan in normal mouse serum was determined

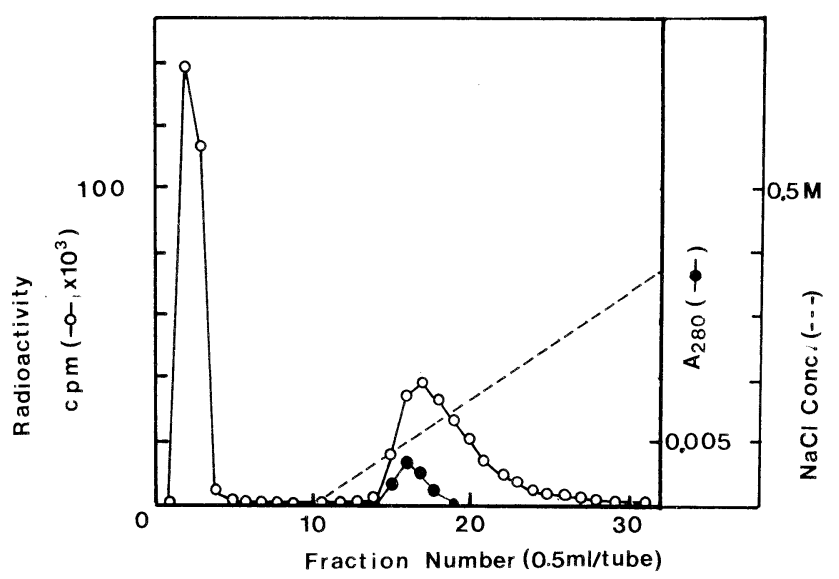

Fig. 1. Anion exchange column (Mono Q) chromatography of tritium-labeled antitumor $\beta$-D-glucan. $\bigcirc$, radioactivity; $\odot$, absorption at $280 \mathrm{~nm}$; ----, salt gradient. 


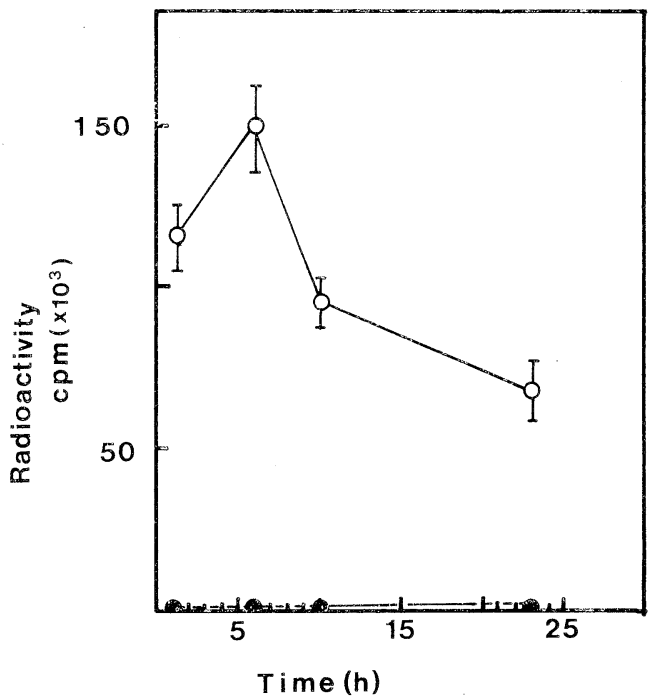

Fig. 2. Radioactivity level in mouse serum after labeled glucan injection ( $\boldsymbol{\Phi}, \mathrm{SD})$. $\bigcirc$, labeled $\beta$-D-glucan; $\bullet$, labeled $\alpha$-D-glucan.

in comparison with that of labeled $\alpha$-1,3-linked D-glucan. The labeled $\alpha$-D-glucan was prepared from branched $\alpha$-D-glucan of Streptococcus mutans OMZ 176 (10) by the same method as that for branched $\beta-1,3-\mathrm{D}$-glucan. Figure 2 shows the change of radioactive levels in the mouse sera after labeled $\beta$-D- and $\alpha$-D-glucan injections. In the case of $\beta$-D-glucan injection into normal mouse, the radioactivity reached a maximum level $6 \mathrm{~h}$ after injection, and gradually decreased. It is interesting that 23 $\mathrm{h}$ after injection the radioactivity level was still high (about half of the maximum level). In contrast to the $\beta$-D-glucan, no radioactivity was detected even $2 \mathrm{~h}$ after the labeled $\alpha$-D-glucan was injected. This rapid clearance of labeled $\alpha$-D-glucan from the serum might result from the susceptibility of the $\alpha$-D-glucan to metabolizing enzymes, such as liver amylases, in mouse organs. These facts suggest that antitumor branched $\beta$-D-glucan is hardly cleared from the mouse serum, probably because of its resistance to carbohydrate metabolizing enzymes. Gel-permeation chromatography of the serum obtained 10 or $23 \mathrm{~h}$ after injection of labeled $\beta$-D-glucan showed that radioactivities in the serum were eluted at the void volume of the column with a minor UV absorption peak which was different from the main UV absorption peak due to the serum proteins (data not shown). These results indicate that no marked degradation of the T-labeled $\beta$-D-glucan occurred or that the glucan was conjugated to some macromolecules such as proteins.

The mouse serum obtained $10 \mathrm{~h}$ after the antitumor $\beta$-D-glucan injection (at a dose of $100 \mathrm{mg} / \mathrm{kg}$ ) showed tumor-regression activity when it was injected into tumor-bearing mice intravenously (11). To determine the relation between the 


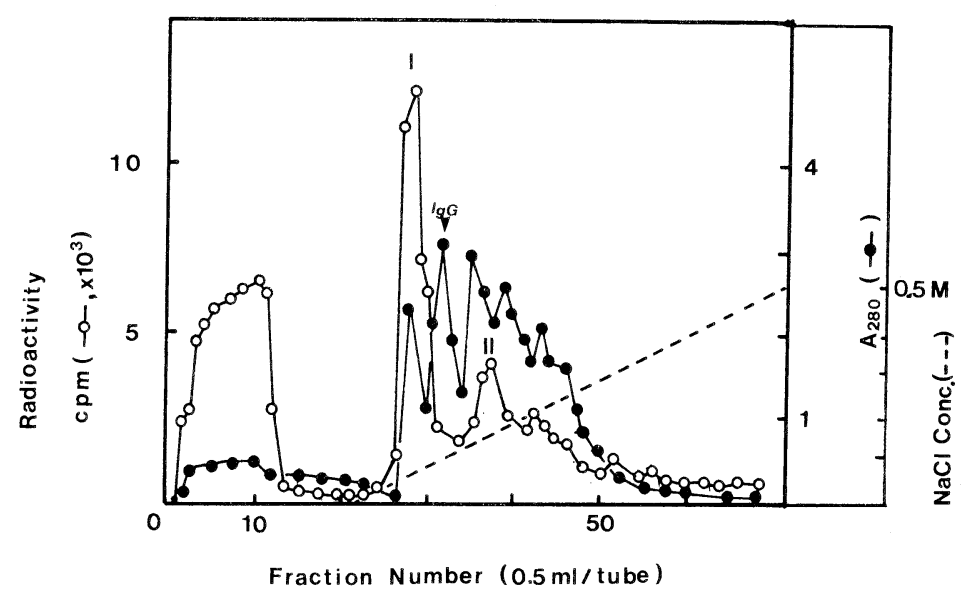

Fig. 3. Anion exchange column (Mono Q) chromatography of mouse serum obtained $10 \mathrm{~h}$ after injection of labeled $\beta$-D-glucan. $\bigcirc$, radioactivity; $\odot$, absorption at $280 \mathrm{~nm} ;-\cdots$, salt gradient.

tumor-regression activity in the serum and the glucan injected, sera were prepared from tumor-bearing mice by the same method as that described previously (6) using T-labeled $\beta$-D-glucan instead of the cold $\beta$-D-glucan. After confirmation of the tumor-regression activity of the mouse serum thus obtained, the serum was subjected to ion-exchange chromatography. Figure 3 shows a profile of ionexchange FPLC using Mono $Q$ h 5/5 column. It is apparent that radioactivity was separated into three fractions, namely, a non-adsorbed fraction, an adsorbed fraction which could be eluted by low $\mathrm{NaCl}$ concentration (peak I) and an adsorbed fraction eluted by high $\mathrm{NaCl}$ concentration (peak II). The peak UV absorption (indicated by an arrow), which appeared between peak I and II, was proved to be mouse immunoglobulin $\mathrm{G}$ (IgG) by immunodiffusion test using anti-mouse IgG antiserum. From the viewpoint of alteration of the properties of the glucan, it is interesting that more than $50 \%$ of radioactive glucan was adsorbed on a Mono $\mathrm{Q}$ anion exchange column. As described above, the T-labeled $\beta-\mathrm{D}$ glucan had been purified as a non-adsorbed glucan fraction on Mono-Q column, and this property of the glucan did not change spontaneously. Taking these facts into account, the result of anion-exchange FPLC suggests that the antitumor glucan acquired the property of adhering to the anion exchange column.

To relate these serum fractions to the tumor-regression activity, each fraction (fraction numbers 9-10, 28, and 36-38 for non-adsorbed, I, and II fraction, respectively) was prepared from $6 \mathrm{ml}$ of mouse serum (collected and pooled from 5 mice) and subjected to the in vivo antitumor assay by their i.v. injections into tumor-bearing mice. This assay showed that only fraction I, which was adsorbed on a Mono $\mathrm{Q}$ column and was eluted by low salt concentration, had the tumor- 
regression activity (inhibition ratio of $73.2 \% 5$ weeks after tumor inoculation). This fact confirms our previous result obtained in experiments using the antibranched $\beta$-1,3-glucan antibody conjugated immunoadsorbent column (6). In that experiment, the tumor-regression factor in the serum, which was obtained by injection of the antitumor $\beta$-glucan, was mostly retained on the immunoadsorbent column. These results suggest that the tumor-regression factor is closely related to the modified glucan or glucan conjugate formed during its circulation in mouse organs. In this connection, Nitta suggested the tumor-regression factor found in mouse serum to be glycoprotein or protein in nature (12).

In conclusion, the facts described herein indicate that the tumor-regression activity found in mouse serum after glucan injection may be due to modified products of the glucan injected.

\section{REFERENCES}

1) Whistler, R. L., Bushway, A. A., and Singh, P. P. (1976): Noncytotoxic antitumor polysaccharides. Adv. Carbohydr. Chem. Biochem., 32, 235-275.

2) Sone, Y., Kakuta, M., and Misaki, A. (1978): Isolation and characterization of polysaccharides of "Kikurage," fruit body of Auricularia auricula-judae. Agric. Biol. Chem., 42, 417-423.

3) Sone, Y., Okuda, R., Wada, N., Kishida, E., and Misaki, A. (1985): Structures and antitumor activities of the polysaccharides isolated from fruiting body and the growing culture of mycelium of Ganoderma lucidum. Agric. Biol. Chem., 49, 2641-2653.

4) Misaki, A., Nasu, M., Sone, Y., Kishida, E., and Kinoshita, C. (1986): Comparison of structure and antitumor activity of polysaccharides isolated from Fukurotake, the fruiting body of Volvariella volvacea. Agric. Biol. Chem., 50, 2171-2183.

5) Kishida, E., Sone, Y., and Misaki, A. (1989): Purification of an antitumor-active, branched $(1 \rightarrow 3)-\beta$-D-glucan from Volvariella volvacea, and elucidation of its fine structure. Carbohydr. Res., 193, 227-239.

6) Kishida, E., Sone, Y., Shibata, S., and Misaki, A. (1989): Preparation and immunochemical characterization of antibody to branched $\beta-(1 \rightarrow 3)$-D-glucan of Volvariella volvacea, and its use in studies of antitumor actions. Agric. Biol. Chem., 53, 18491859.

7) Sasaki, T., Tanaka, M., and Uchida, H. (1982): Effect of serum from mice treated with antitumor polysaccharide on expression of cytotoxicity by mouse peritoneal macrophages. J. Pharm. Dyn., 5, 1012-1016.

8) Doubois, M., Gilles, K. A., Hamilton, J. K., Rebers, P. A., and Smith, F. (1956): Colorimetric method for determination of sugars and related substances. Anal. Chem., 28, 350-356.

9) Kishida, E., Sone, Y., and Misaki, A. (1992): Effects of branch distribution and chemical modifications of antitumor $(1 \rightarrow 3)-\beta$-D-glucan. Carbohydr. Polymers, 17, 8995.

10) Ebisu, S., Misaki, A., Kato, K., and Kotani, S. (1974): The structure of waterinsoluble glucan of cariogenic Streptococcus mutans, formed in the absence and presence of dextranase. Carbohydr. Res., 38, 374-386. 
11) Sone, Y., Shibata, S., Kishida, E., and Misaki, A. (1987): Mechanism of antitumor action of branched $\beta$-(1 $\rightarrow 3)$-D-glucan. Seikagaku, 59, 787.

12) Nitta, K. (1983): Mechanism of action of antitumor polysaccharides. Annals Soc. BCG Immunother., 7, 1-12. 